\title{
Corrigendum to "A New Approach to Increase the Flexibility of Curves and Regular Surfaces Produced by 4-Point Ternary Subdivision Scheme"
}

\author{
Rabia Hameed $\mathbb{D}^{\mathbb{D}},{ }^{1}$ Ghulam Mustafa $\mathbb{D}^{2},{ }^{2}$ Amina Liaqat $\mathbb{D}^{1},{ }^{1}$ Dumitru Baleanu $\mathbb{D},{ }^{3,4,5}$ \\ Faheem Khan $\left(\oplus^{6}{ }^{6}\right.$ Maysaa M. Al-Qurashi $i{ }^{7},{ }^{7}$ and Yu-Ming Chu $\oplus^{8,9}$ \\ ${ }^{1}$ Department of Mathematics, The Government Sadiq College Women University Bahawalpur, Bahawalpur, Pakistan \\ ${ }^{2}$ Department of Mathematics, The Islamia University of Bahawalpur, Bahawalpur, Pakistan \\ ${ }^{3}$ Department of Mathematics, Cankaya University, Ankara 06530, Turkey \\ ${ }^{4}$ Institute of Space Sciences, Magurele-Bucharest 077125, Romania \\ ${ }^{5}$ Department of Medical Research, China Medical University Hospital, China Medical University, Taichung 40402, Taiwan \\ ${ }^{6}$ Department of Mathematics, University of Sargodha, Sargodha, Pakistan \\ ${ }^{7}$ Department of Mathematics, Faculty of Sciences, King Saud University, Riyadh 11451, Saudi Arabia \\ ${ }^{8}$ Department of Mathematics, Huzhou University, Huzhou 313000, China \\ ${ }^{9}$ Hunan Provincial Key Laboratory of Mathematical Modeling and Analysis in Engineering, \\ Changsha University of Science and Technology, Changsha 410114, China
}

Correspondence should be addressed to Yu-Ming Chu; chuyuming2005@126.com

Received 2 February 2021; Accepted 2 February 2021; Published 20 February 2021

Copyright ( 92021 Rabia Hameed et al. This is an open access article distributed under the Creative Commons Attribution License, which permits unrestricted use, distribution, and reproduction in any medium, provided the original work is properly cited.

In the article titled "A New Approach to Increase the Flexibility of Curves and Regular Surfaces Produced by 4Point Ternary Subdivision Scheme" [1], authors "Faheem Khan" and "Maysaa M. Al-Qurashi" had incorrect affiliations.

Faheem Khan was incorrectly affiliated to Department of Mathematics, Faculty of Sciences, King Saud University, Riyadh 11451, Saudi Arabia.

Maysaa M. Al-Qurashi was incorrectly affiliated to Department of Mathematics, University of Sargodha, Sargodha, Pakistan.

The correct affiliations for the authors are as follows.

Faheem Khan-Department of Mathematics, University of Sargodha, Sargodha, Pakistan.

Maysaa M. Al-Qurashi-Department of Mathematics, Faculty of Sciences, King Saud University, Riyadh 11451, Saudi Arabia.

The corrected list of affiliations is shown in the author information above.

\section{References}

[1] R. Hameed, G. Mustafa, A. Liaqat et al., “A New Approach to Increase the Flexibility of Curves and Regular Surfaces Produced by 4-Point Ternary Subdivision Scheme," Mathematical Problems in Engineering, vol. 2020, Article ID 6096545, 17 pages, 2020. 